CERN-PPE/96-136

2 October 1996

\title{
FELIX, a full acceptance detector for the CERN LHC
}

\author{
Karsten Eggert, \\ CERN, Geneva, Switzerland \\ Cyrus Taylor \\ Dept. of Physics, Case Western Reserve University, Cleveland, OH 44106 USA
}

\begin{abstract}
Plans for FELIX, a full acceptance detector for the future CERN Large Hadron Collider (LHC), are described. The physics goals include detailed studies of the strong interactions (QCD), the forward energy flow and diffractive processes (Pomeron interactions), electroweak rapidity gaps, and elastic scattering. The precise measurement of particle production at a centre-of-mass energy of $14 \mathrm{TeV}$, well above the cosmic ray "knee", will be fundamental for the interpretation of the highest energy cosmic ray events and may clarify some of the anomalies indicated by some cosmic ray experiments. The magnetic architecture and some of the design issues are briefly reviewed.
\end{abstract}

(Submitted to Nuclear Physics) 
The experimental program of the future Large Hadron Collider (LHC) at CERN, as currently envisaged, has three components. The approved experiments, ATLAS [1] and CMS [2], running at the highest possible luminosity of $10^{34} \mathrm{~cm}^{-2} \mathrm{~s}^{-1}$, will concentrate on rare, large transverse momentum phenomena in the central rapidity region. Two further experiments have been proposed but have not yet received final approval. LHC-B [3] will study CP violation and B decays. ALICE [4] will study heavy ion collisions.

It has, however, been recognized for some time that the next generation of hadron-hadron colliders should fully exploit the intrinsic diversity of high energy hadron collisions [5]. FELIX, a full acceptance detector that will be capable of measuring almost completely the energy- and particle- flow in pp collisions over the entire kinematic range down to zero degree production angles will thus pursue a program of physics complementary to that of the other planned experiments [6]. At the most basic level, the argument for such a detector notes that interesting physics is kinematically allowed for all pseudorapidities $|\eta|<\sim 10$. This is illustrated in Fig. 1 in which the charged particle density (photons are similar) and the energy flow, as predicted by Pythia [7], are plotted as a function of the pseudorapidity. It is obvious that the proposed detectors such as ATLAS and CMS will miss most of the energy in generic pp collisions. We thus regard the case for a full acceptance detector as compelling, particularly since previous colliders (ISR, SPS, FNAL) were also deficient in this respect.

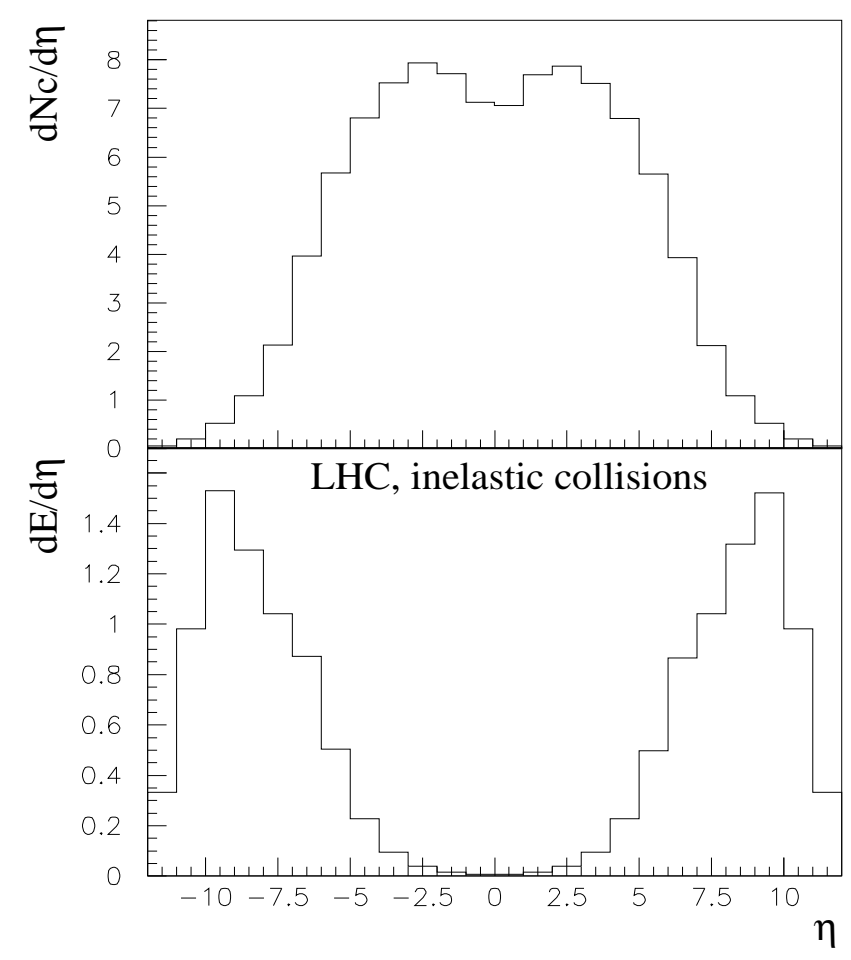

Figure 1: Pseudorapidity distribution of charge particles and of the energy-flow.

\section{PHYSICS GOALS}

A full acceptance detector will maximize the ability to search for new physics in unexplored regions of phase space while pursuing a substantial agenda of more conventional physics. Many of these topics are not well understood, either theoretically or experimentally, and we be- 
lieve that the prospect of acquiring data will serve as a powerful stimulus to the further development of these topics.

The physics agenda for FELIX will thus include the complete range of non-perturbative and semi-perturbative QCD processes which are of considerable intrinsic interest, and which appear as backgrounds to other interesting and potential new physics.

At the lowest luminosity we will concentrate on soft physics such as inclusive distributions of forward and very forward reaction products and leading particle effects. The data will enable us to search for disoriented chiral condensates and other related phenomena by studying multiple particle correlations.

Particle beams can be defined via leading particle tags and meson-nucleon interactions at energies of several TeV can be observed. FELIX will have the unique capability to see leading particles, with $0.1<x_{F}<0.99$. If a leading nucleon or delta is detected, the remainder of the event represents a collision of a beam nucleon with the exchanged nonstrange meson; hence the LHC becomes an effective meson-nucleon collider, and most pp processes studied, hard or soft, can be compared with the corresponding meson-proton process to determine the beam-composition dependence. A kaon "beam" can be made in a like manner with a leading lambda tag.

The physics agenda continues with the study of soft single and double diffraction (Pomeron exchange) and of Pomeron-Pomeron interactions via triple and higher order diffraction. FELIX will measure the ultimate diffractive process-elastic scattering-over a wide range of momentum transfers: small enough to determine the total cross-section and large enough to see the dip structure and the distribution beyond the dip.

Increasing the luminosity by an order-of-magnitude to some $10^{31} \mathrm{~cm}^{-2} \mathrm{~s}^{-1}$ will lead us to semi-hard and hard diffractive physics. There is much current interest in the nature of the "hard Pomeron" mediating these processes, which, it is hoped, can be quantitatively understood in terms of a theoretical construct arising from multi-peripheral gluon exchange. We will continue the studies already begun at HERA, Fermilab and the CERN SPS collider. More generally, the structure of the Pomeron will be elucidated through the measurement of the rapidity distributions of jets, heavy flavors and gauge bosons. Related studies, probing the fractal nature of QCD phase space, and the onset of color-coherence effects, include the analysis of multi-jet global patterns and of jets-within-jets.

Rapidity gap tags may provide a useful tool in the search for new physics. For example, Pomeron-Pomeron interactions may be an effective way of producing new particles in a rather clean environment, particularly if the Pomeron has a hard gluon structure function. Further, while rapidity gaps are usually thought of as a consequence of a strongly interacting color singlet (the pomeron or hard pomeron), hard collisions mediated by the exchange of gauge bosons can also create rapidity gaps. Together with the expectation that hard diffractive processes may be enhanced by orders-of-magnitude compared to the most naive two-gluon exchange mechanism if the parton-parton center of mass momentum is sufficiently high, we are led to the study of two gauge-boson physics, new physics and diffractive production of heavy flavors via rapidity-gap tags.

The measurement of forward reaction products and leading particle effects will be of utmost importance in interpreting the highest energy cosmic ray air showers. Not much is known about the energy flow in the far-forward region and the interpretation of high energy cosmic ray shower is mainly based on some forward production models in the Monte-Carlo calculations of extensive air showers. Furthermore, a variety of cosmic ray exotica, such as the knee in the cosmic ray spectrum at a centre-of-mass energy of about $5 \mathrm{TeV}$, Centauro and Anti-Centauro events, forward multi-muon bundles, aligned clusters and possible changes in the interaction length may 
find a natural explanation or may be confirmed if the energy flow in the far-forward region is better understood.

We want to emphasize the importance of studying proton-ion collisions which could become available due to the new design in which the RF cavities are split into two, each for one ring. The present extensive program of A-dependence physics, centered on shadowing and colortransparency phenomena, could be extended to these extreme collider energies. In addition, it may be that any kind of exotic physics which is associated with beam fragmentation into a very high mass system (e.g. Centauro, anti-Centauro, disoriented chiral condensate) occurs with higher frequency in proton-ion collisions than in proton-proton collisions.

Finally, the detector we envision should also be well-suited to two photon physics in pp and heavy ion collisions, opening a new field of photoproduction physics (pioneered by HERA), and searches for new massive particles and multi-quark states. Intense heavy-ion beams represent a prolific source of quasireal photons and hence reasonable statistics can be obtained at two-photon masses well above the reach of LEP 200.

\section{THE FELIX SET-UP}

The basic philosophy of our detector is that it will be a survey instrument: sensitive to almost everything, and optimized for almost nothing, i.e. a detector with good capabilities to see the unexpected as well as "engineered" discoveries. We note that some of the items on the physics agenda place stringent requirements on the detector. For example, the analysis of events with rapidity gaps requires coverage without holes over the maximum range of pseudorapidity attainable, while studies of jets-within-jets requires fine segmentation. Studies of disoriented chiral condensates require the ability to simultaneously see both charged particles and photons. The challenge is to design a detector which can meet all these requirements in the difficult environment of forward physics at a hadron collider.

The strength of a full acceptance detector will be in the perception of complicated patterns in individual events. This leads essentially to the following design goals:

- all charged particles and photons of generic $p_{t}$ should be observed and their momenta wellmeasured over the entire phase space (both arms)

- the physics of rapidity gaps should not be compromised

- the granularity of the detectors should be sufficient to resolve multi-TeV jets

- diffractive and elastically scattered protons should be well-measured

- identification of muons should be extended into the far-forward regions.

Furthermore, the overall cost of the experiment has to be minimized by the use of existing material, e.g. magnets, and by an optimal use of the interaction region, including caverns and infrastructure.

Fig. 2 is a sketch of the central and the very forward part of FELIX in the intersection region I4, which can be equipped with an insertion well suited for a forward experiment. The basic magnetic architecture is given by the central ALEPH solenoid (1.6 Tesla), already in place, complemented on both sides by dipole magnets with a horizontal field of 0.7 - 1 Tesla. These magnets, excited by superconducting coils, can be easily constructed out of the modular UA1 magnet. Since the experiment has to extend well into the forward beam lines it is important that the magnetic architecture of the experiment is fully integrated into the design of the machine lattice. The two horizontal dipoles UA1 and D0 provide the vertical crossing-angle of about 0.6 mrad needed to avoid long range tune shift effects at large $\beta$-values. The vertical dipoles D1 (split into three magnets) and D2 (two magnets) separate the beams horizontally to the required distance of $42 \mathrm{~cm}$. All forward magnets (D0,D1 and D2) with a free bore of $10 \mathrm{~cm}$ radius and a field strength of 4 Tesla are identical in their design (taken from RHIC) and differ only in their 


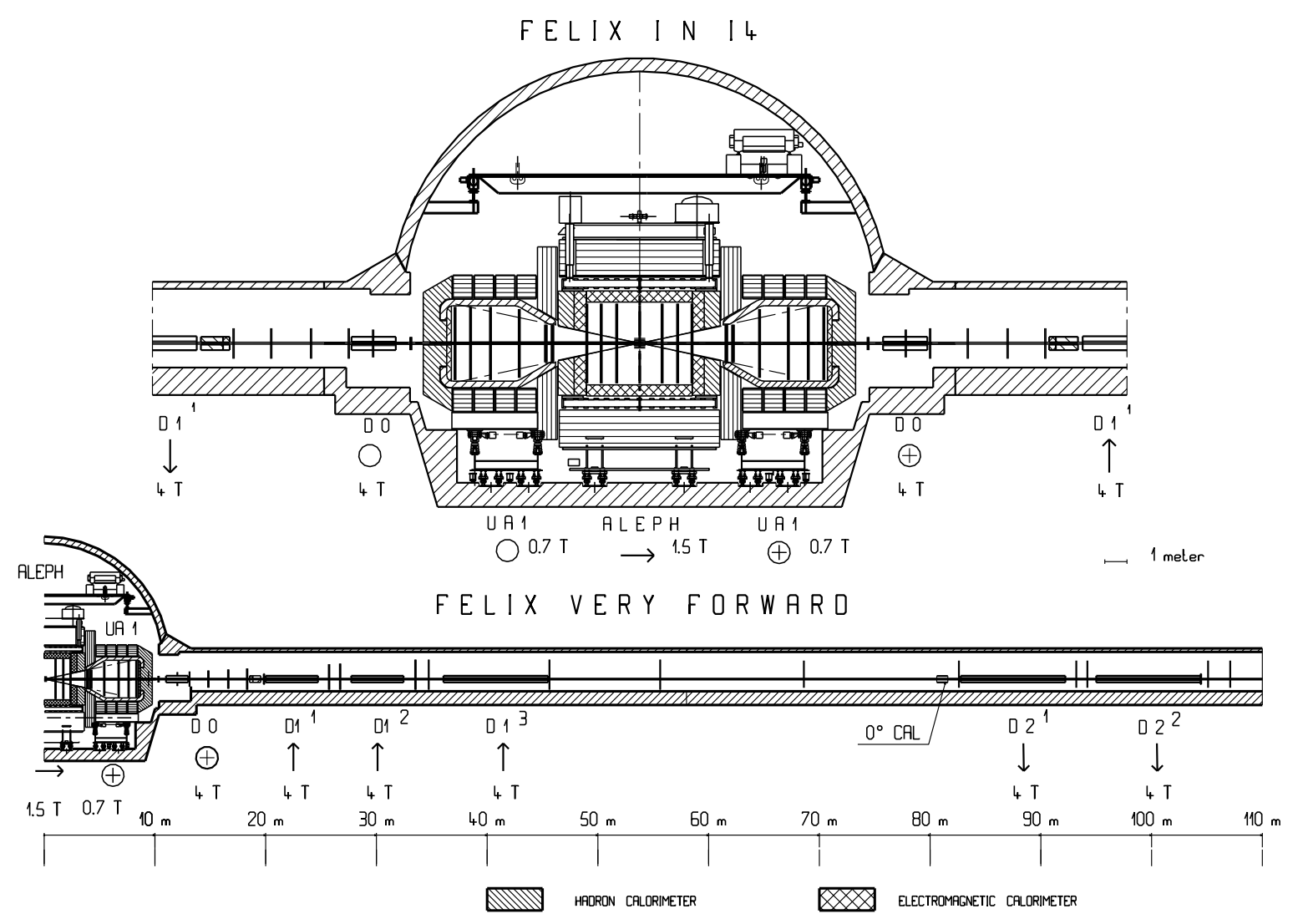

Figure 2: Sketch of the FELIX experiment at I4 (central and very forward region).

length. Thus the forward magnets are used to guide the beams and at the same time they serve as powerful spectrometer magnets.

The measurements of very forward particles are especially difficult since they have to be performed before the particles shower in the surrounding material. The vacuum-chamber architecture, the calorimetry close to the beams, and the implementation of "Roman pots" have to be optimized and thus present a major challenge. Forward particles are bent out of the beam profile according to their momenta in the different magnets and their momenta are measured with detectors placed as close as $2 \mathrm{~cm}$ away from the beam. Thus each forward spectrometer covers a certain range in momentum. The spectrometers are designed so that they have overlapping acceptances. Thus a charged particle track is acquired in one magnet and the measurement of it's momentum is improved in the following one. As a consequence of the larger bending power of the very forward magnets the momentum resolution stays well below $0.5 \%$ up to the beam momentum (assuming a spatial resolution of $50 \mu \mathrm{m}$ per detector plane).

For the detectors close to the beam we envisage a combination of pixel detectors with $0.1 \mathrm{~mm}$ pixel size (for pattern recognition) and silicon strip detectors (for resolution improvement).

The design of the beam pipe plays an important role in the optimization of the very forward detector. It has to be as transparent as possible to avoid particle showering, but it should also permit the placement of detectors close to the beam. Hence large diameter vacuum pipes can only be considered if a radio-frequency screen around the beam minimizes electrical losses and disturbences to the beams.

The charged particle measurements will be complemented by complete electromagnetic 
and hadronic calorimetric coverage extending down to zero degrees. Such a hermetic detector will, for the first time, provide a measurement of total missing energy, a powerful tool in the search for new physics. The interplay between calorimetry and charged particle tracking is delicate, however. In principle, charged particle momenta should be measured by tracking before they enter the calorimeters. This is complicated, however, because each stage of calorimetry also defines the aperture restriction for subsequent stages of tracking. Thus care is needed to avoid splash and leakage from the calorimeters interfering with subsequent stages of tracking. Detailed GEANT simulations are in progress to help optimize the forward detectors, and to determine the occupancy of the forward trackers and the granularity of the calorimeters.

Finally, an almost complete muon coverage will extend down close to the beams.

We have devoted comparatively little effort to detailed work on the detector architecture in the central region. Unlike the forward direction, the technical challenges in the central region are reasonably well understood, and we will be able to profit from the experience of the many existing or planned central detectors.

FELIX will run at a reduced luminosity of $10^{30}-10^{32} \mathrm{~cm}^{-2} \mathrm{sec}^{-1}$ while the other experiments are at full luminosity. In order to reduce the luminosity locally in 44 by up to three orders of magnitude relative to the other experiments, and to minimize the beam divergence for the elastic scattering measurement, $\beta$-values at the intersection point (IP) of up to $1000 \mathrm{~m}$ or more have to be achieved. Fortunately the group of T. Taylor (CERN-LHC) has designed a new insertion for I4 (Fig. 3) which takes into account the FELIX experimental magnets together with the constraint that the beams be separated by $42 \mathrm{~cm}$ at the location of the RF cavities [8].

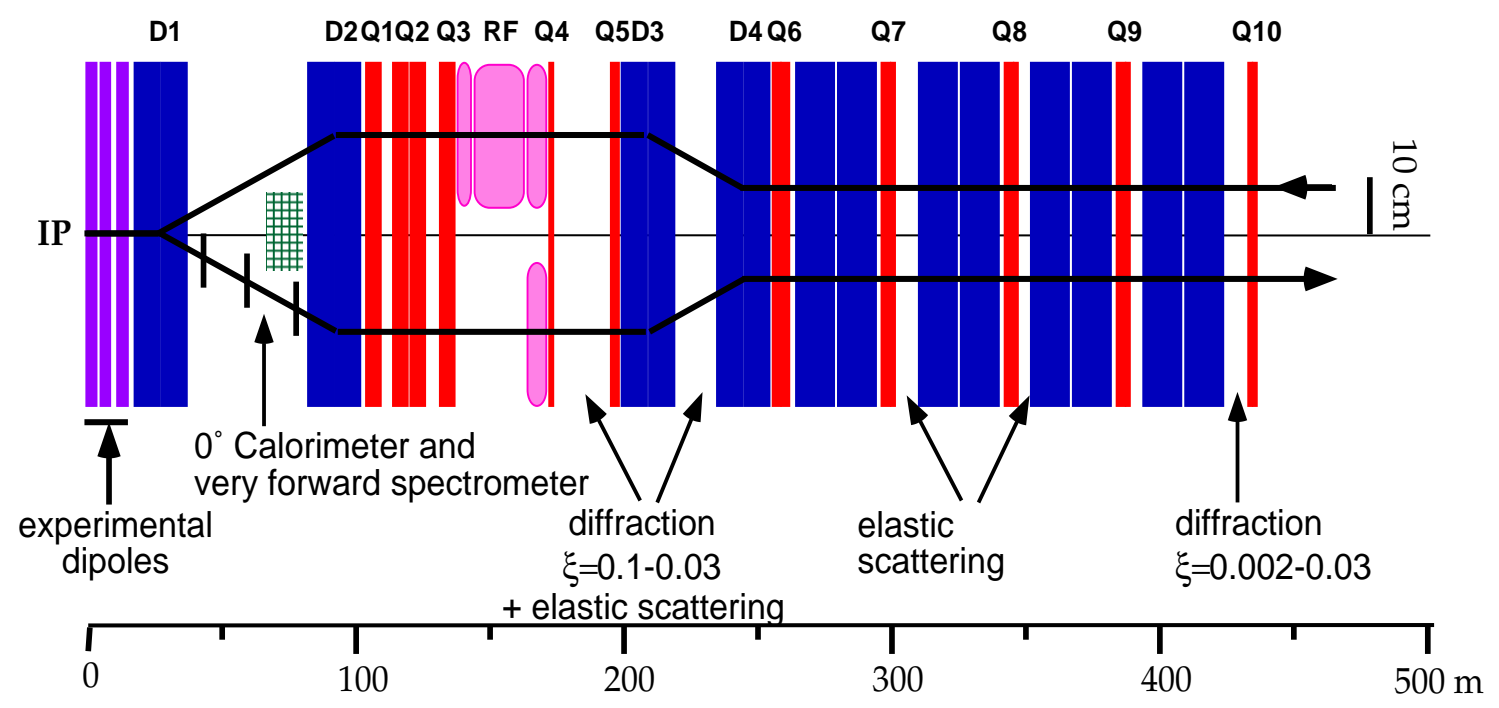

Figure 3: The proposed insertion at I4, also indicating the positions of the "Roman pots" upstream.

The basic feature of the design is that the inner quadrupole triplet is located behind the beam separating magnets, leaving sufficient space between the magnets D1 and D2 for the very forward spectrometer and the zero degree calorimeter. Further, the proposed insertion can be tuned so that the $\beta$-value in IP4 is either in the range of $4 m<\beta^{*}<30 m$ or $300 m<$ $\beta^{*}<1500 \mathrm{~m}$. While precluding reaching the very highest luminosities of the major detectors $\left(\beta^{*}=0.5 \mathrm{~m}\right)$, this is nicely matched to the FELIX physics goals. After completion of the lowluminosity running necessary to achieve the physics goals outlined here, it should be possible to modify the insertion in order to achieve lower values of $\beta^{*}$. 

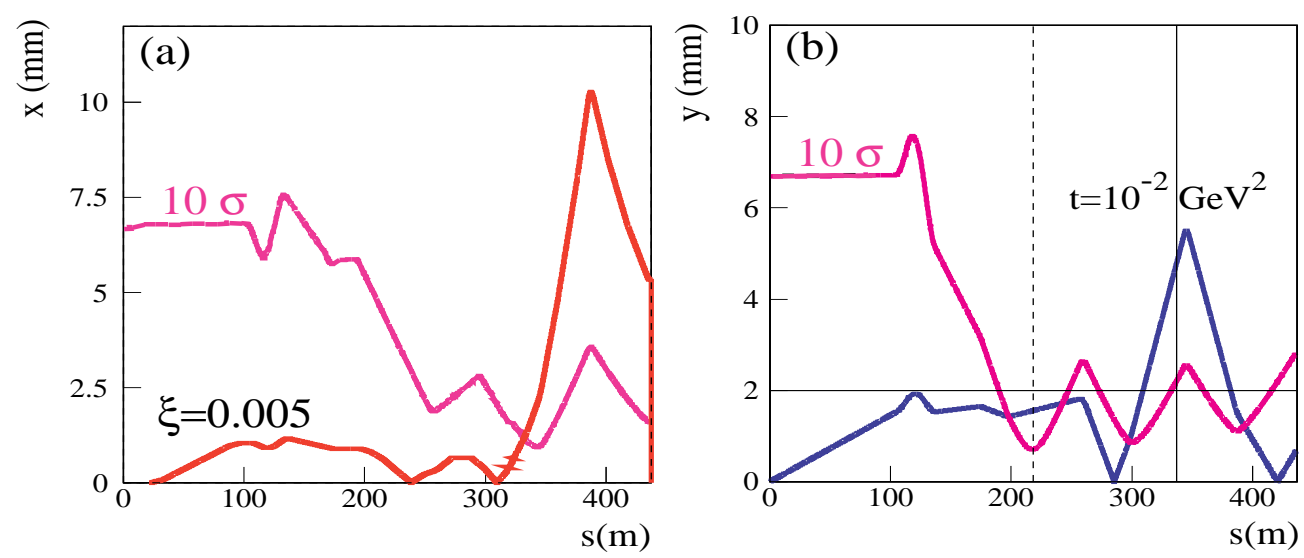

Figure 4: The $10 \sigma$ beam profile as a function of the distance from the IP and the excursion of (a) a diffractive proton $(\xi=0.005)$; (b) an elastically scattered proton $\left(t=10^{-2} \mathrm{GeV}^{2}\right)$.

FELIX has to extend several hundred meters upstream in order to measure diffractive and elastically scattered protons. The detectors are placed in "Roman" pots so that they can be moved as close to the beam as possible (probably $10 \sigma$ ) during stable beam conditions, and be retracted during beam injection and acceleration. The optimized locations of these tracking stations are indicated in Fig. 3. Fig. 4 plots the $10 \sigma$ beam profile for the two directions perpendicular to the beam ( $x$ lies in the beam-bend plane) for a $\beta^{*}=\beta(0)=1000 \mathrm{~m}$. For $s>200 \mathrm{~m}$, the $10 \sigma$ beam width is around $2 \mathrm{~mm}$. An elastically scattered proton with $t<\sim 10^{-2} \mathrm{GeV}^{-2}$ can be measured well due to it's excursion of about $6 \mathrm{~mm}$ (Fig. 4(b)). The excursion of a diffractive proton with a relative momentum loss $\xi=\Delta p / p=0.5 \%$ is largest at $s \approx 350 \mathrm{~m}$, where the dispersion of the machine is about $2 \mathrm{~m}$ (see Fig. 4(a)). It should be possible to measure diffractive protons with $\xi>\sim .2 \%$.

\section{CONCLUDING REMARKS}

FELIX will provide an exciting and powerful new tool for the study of physics at the LHC. While low in cost compared to the major detectors, such a full acceptance detector will combine a strong program of physics complementary to those of the other detectors with a substantial discovery potential, particularly for the "unexpected".

\section{References}

[1] ATLAS collaboration, "Letter of Intent for a General-Purpose pp Experiment at the Large Hadron Collider at CER", CERN/LHCC 92-4, (1 Oct. 1992).

[2] CMS collaboration, "CMS Letter of Intent", CERN/LHCC 92-3, (1 Oct. 1992).

[3] LHC-B collaboration, "A Dedicated LHC Collider Beauty Experiment for Precision Measurements of CP-Violation”, CERN/LHCC 95-5, (25 Aug. 1995).

[4] ALICE collaboration, "Letter of Intent for A Large Ion Collider Experiment", CERN/LHCC 93-16, (1 March 1993).

[5] J. D. Bjorken, Int. J. Mod. Phys. A7 (1992) 4189.

[6] See the FELIX www pages at http://www.cern.ch/FELIX

[7] T. Sjostrand, Comp. Phys. Comm. 92 (1994) 74.

[8] T. Taylor and R. Ostijic, private communication. 